\title{
Internal shocks model for microquasar jets
}

\section{Omar Jamil ${ }^{* i}$}

University Of Southampton

E-mail: 0 .jamilephys.soton.ac.uk

\section{Rob Fender, Christian Kaiser}

University Of Southampton

\begin{abstract}
We present an internal shocks model to investigate particle acceleration and radiation production in microquasar jets. The jet is modelled with discrete ejecta at various time intervals. These ejecta (or 'shells') may have different properties including the bulk velocity. Faster shells can catch up and collide with the slower ones, thus giving rise to shocks. The particles are accelerated inside the shocked plasma. Each collision results in a new shell, which may take part in any subsequent collisions as well as radiate due to synchrotron radiation. Almost continuous energy dissipation along the jet can be obtained with a large number of shell collisions. We investigate the spectral energy distribution of such jets as well as the physical significance of various parameters (e.g. the time interval between ejections and the shell size)
\end{abstract}

VII Microquasar Workshop: Microquasars and Beyond September 1-5 2008

Foca, Izmir, Turkey

\footnotetext{
* Speaker.

${ }^{\dagger}$ Grateful to STFC for the Ph.D. funding
} 


\section{Introduction}

Jets are observed from a large variety of astronomical objects, ranging from young stellar objects (YSO) to active galactic nuclei (AGN). In terms of emission, flat radio ( $\alpha=0$ when $F_{v} \propto$ $v^{\alpha}$ ) spectra have commonly been observed from these sources (AGN and X-ray binaries (XRB)). Observations of XRBs suggest a frequency independent flat spectrum extending down to millimetre [4] or even near-infrared bands [3]. Flat radio spectra from AGN and XRBs have traditionally been attributed to partially self-absorbed jet emission from the innermost region of a conical jet [1], [5]. However, in order to obtain such flat spectra from partially self absorbed synchrotron emission we either need continuous re-acceleration all along the emitting jet region or use a non conical jet model [6]. If we assume a conical geometry for jets then the acceleration mechanisms problem needs to be addressed. We explore internal shocks as a re-acceleration mechanism for microquasar jets.

\section{The model}

Our model has been inspired by the internal shocks model for radio-loud quasars [8]. We assume a conical geometry for the jet with a given full opening angle $\theta_{e}$. Plasma is injected into the jet in the form of discrete packets or "shells".

\subsection{Shell properties}

Our model is heavily based on Spada et al. model [8] with a few changes in order to adapt the model to XRBs. The injected shell properties determine most of the observables for the simulation. The central engine for shell ejections is considered to be intermittent in nature, with a time interval $\left(\triangle_{t}\right)$ between ejections. Each shell also has a given bulk Lorentz factor (BLF), $\Gamma$, associated with it. Outside of the collisions, the $\Gamma$ does not evolve as a shell moves through the jet. In order to choose $\Gamma$ for a given shell we set the minimum $\left(\Gamma_{\min } \gtrsim 1\right)$ and maximum $\left(\Gamma_{\max }\right) \Gamma$ and then sample randomly from that range.

The shell mass is determined by the combination of kinetic luminosity of the jet $\left(L_{W}\right)$, the time jet is on for $\left(t_{j e t}\right)$ and the total number of shells to be injected during that time $(N)$. The following relation sets the total mass in the jet:

$$
\sum_{j=1}^{N} M_{j} \Gamma_{j} c^{2}=L_{W} t_{j e t},
$$

where $c$ is the velocity of light and $M_{j}$ is the mass of an arbitrary shell. However, as we have assumed the central ejection engine to be intermittent in nature and accumulative, the time gap between ejections simply accumulates more mass for a given shell.

$$
M_{j}=\frac{L_{W} \triangle t_{j}}{\Gamma_{j} c^{2}},
$$

where $\triangle t_{j}$ denotes the time interval between ejections $j-1$ and $j$. We also set the width of the shell $\left(\triangle_{j}\right)$. 
In order to calculate the time gap between ejections, we take a Gaussian distribution with the mean corresponding to the mean time gap $\left(\triangle t_{j}\right)$ we desire. We then sample from that distribution with chosen standard deviation. If the standard deviation is set to be low then the average time gap between ejections will be close to the mean and the total number of shells to be injected will be $\approx t_{j e t} / \triangle_{t}$. With a larger standard deviation, the total number shells to injected will deviate from the previously stated relation. The variations in the ejection time gap as well as the bulk Lorentz factors of the shells leads to various shells catching up with each other, causing collision.

\subsection{Two-shell collisions}

Once the above parameters are determined, we can set the injection times as well as all the properties of the shells at the start of the simulation. With the variations in $\Gamma$ and $\triangle t_{j}$ we can have a large number of collisions taking place. The time gap between any two collision is:

$$
d t_{c o l}=\frac{R_{(j-1)}^{\text {inner }}-R_{j}^{\text {outer }}}{\left[\beta_{(j-1)}-\beta_{j}\right] c+0.5\left[\beta_{(j-1)}^{e}+\beta_{j}^{e}\right] c},
$$

where $R^{\text {inner }}$ and $R^{\text {outer }}$ are the radii to shell inner (with respect to the source) and outer boundary i.e. the shell has a finite width. $\beta$ is the shell velocity and $\beta^{e}$ is the shell thermal expansion velocity,

$$
\beta^{e}=\frac{2 \beta_{s}^{\prime}}{\Gamma^{2}} \frac{1}{1-\left(\beta \beta_{s}^{\prime}\right)^{2}},
$$

where $\beta_{s} c=v_{s}^{\prime}$ is velocity of sound in the plasma in the shell co-moving frame with

$$
v_{s}=\sqrt{\frac{1}{3} \frac{E_{t h}^{\prime}}{M}}
$$

$E_{t h}$ denotes the thermal energy of the shell, causing the shell to expand in between collisions.

The collisions are treated as inelastic collision. Momentum and energy conservation laws give us the merged shell properties. Merged shell mass is simply given by:

$$
M_{m}=M_{i}+M_{o}
$$

where subscripts $i$ and $o$ denote inner faster and outer slower shell involved in the collision. If we have the interacting shells bulk Lorentz factor, Mass and internal energy $(\eta)$, the merged shell bulk Lorentz factor has the form:

$$
\Gamma_{m}=\left(\frac{\mu_{i} \Gamma_{i}+\mu_{o} \Gamma_{o}}{\mu_{i} / \Gamma_{i}+\mu_{o} / \Gamma_{o}}\right)^{2}
$$

where $\mu_{i}=M_{i}+\eta_{i} / c^{2}$ and $\mu_{o}=M_{o}+\eta_{o} / c^{2}$. The internal energy of the merged shell is given by:

$$
E_{\text {in }}=\eta_{i}+\eta_{o}+\mu_{i} c^{2}\left(\Gamma_{i}-\Gamma_{m}\right)+\mu_{o} c^{2}\left(\Gamma_{o}-\Gamma_{m}\right) .
$$

The merged shell width is calculated by hydrodynamical treatment of the shocked plasma. Adapting the jump equations [2] for forward and reverse shock at the point of collision of the two shells we get the width (in the lab frame) of the outer shock as: 


$$
\triangle_{s h, o}=\frac{\triangle_{o}}{\rho_{o}}
$$

where $\triangle_{o}$ is the outer shell width and

$$
\rho_{o}=\frac{\Gamma_{m}}{\Gamma_{o}} \frac{\hat{\gamma} \Gamma_{o}+1}{\hat{\gamma}-1}
$$

where $\hat{\gamma}$ is the adiabatic index. Equivalent equations stand for the reverse shock involving the inner shell. The width of the two corresponding shocks then gives the width of the newly formed shell. It should be noted that the overall effect of the above treatment is to cause compression in the plasma involved in the collision.

\subsection{Shocked plasma}

The energy generated in the shocked compressed plasma is used in the following three ways (assuming equipartition):

1. Accelerate electrons to a powerlaw distribution.

2. Generate magnetic field for the synchrotron radiation.

3. Thermal expansion of the shells. However, we do not explicitly take thermal electron distribution into account.

The energy split between the above is parameterized in the form of $u_{e}, u_{B}$ and $u_{t h}$. These determine the fraction of the total energy generated that can be made available for the respective process (i.e $\left.u_{e}+u_{B}+u_{t h}=E_{i n t}\right)$. The parameter $u_{t h}$ determines the thermal energy content of a given shell which in turn determines the local sound speed. This is used to calculate the longitudinal (along the jet) expansion velocity of a shell.

\subsection{Powerlaw electron distribution}

With each collision, the electrons are accelerated to a powerlaw distribution of the form:

$$
N(E) \mathrm{d} E=\kappa E^{-p} \mathrm{~d} E
$$

where $E=\gamma m_{e} c^{2}$ is the electron energy and $\kappa$ is the normalization constant (which can be obtained by integrating the above relation between $E_{\min }$ and $E_{\max }$ ). The acceleration is treated as instantaneous and uniform in the respective volume. At each collision even the powerlaw distribution of electrons is completely replaced by a new one. However, in between collisions the powerlaw distribution "ages" via the evolution of $\kappa$ as well as $E_{\max }$.

With the assumption of a conical jet and expanding shells (see 2.2), we need to take adiabatic losses into account as well (see 2.6). 


\subsection{Magnetic field}

Parameter $u_{B}$ determines the fraction of energy available for the magnetic energy $\left(E_{B}=u_{B} \cdot E_{i n t}\right)$ :

$$
E_{B}=\frac{B^{2}}{2 \mu_{0}}
$$

where $\mu_{0}$ is the magnetic permeability. This gives the magnetic field strength available to synchrotron radiation. The magnetic field is assumed to be randomly oriented and tangled in the plasma. This allows us to treat the magnetic field as an ultra-relativistic gas $(\hat{\gamma}=4 / 3)$, giving a straightforward adiabatic loss treatment for the magnetic field.

\subsection{Adiabatic losses}

As we are dealing with expanding shells, we need to take the adiabatic losses into account. The shells expand both laterally due to conical jet as well as longitudinally due to thermal expansion of the shells. Using the treatment outlined in [7], we get a relation for the change in energy due to adiabatic losses

$$
\frac{E}{E_{0}}=\left(\frac{V}{V_{0}}\right)^{-1 / \hat{\gamma}},
$$

This relation can therefore be used for both electron energy losses as well as the magnetic energy losses.

\subsection{Synchrotron spectrum}

The Synchrotron radiation treatment outlined in [7] is employed in our model. As noted earlier, we assume that any given shell moves with constant velocity along the jet, unless it collides. For a shell with bulk Lorentz factor $\Gamma$ and velocity $\beta$ and the jet axis making an angle $\theta$ with the observer, we get the Doppler factor of

$$
\delta_{\mp}=[\Gamma(1 \mp \beta \cos \theta)]^{-1},
$$

where $\mp$ are the receding and approaching jets respectively. This then leads to observable monochromatic intensity

$$
I_{v}=\delta_{\mp}^{3} \frac{J_{v}}{4 \pi \chi_{v}}\left(1-e^{-\chi_{v} r}\right),
$$

where $J_{v}$ is the emissivity per unit volume, $\chi_{v}$ is the absorption coefficient and $r$ is the radius of the shell. The frequency is measured in the rest frame of the jet material. It is related to the observed frequency by $v_{o b s}=\delta_{\mp} v$. With the estimate of distance to the source and equation 2.15, we can calculate the flux density for any given shell.

\section{Results}

As mentioned earlier, the Radio to IR flat spectrum from a conical jet is a commonly accepted picture (for XRBs and AGN), yet not fully explained. Therefore, with our model in its early 
stages of development, obtaining the flat spectrum is quite an interesting diagnostic. However, it is highly non-trivial to obtain a realistic flat spectrum from a conical jet without making certain assumptions about the re-acceleration mechanism. We present some preliminary results that show the self consistently calculated synchrotron spectra obtained from a conical jet with internal shocks taking place along the entire length of the jet.

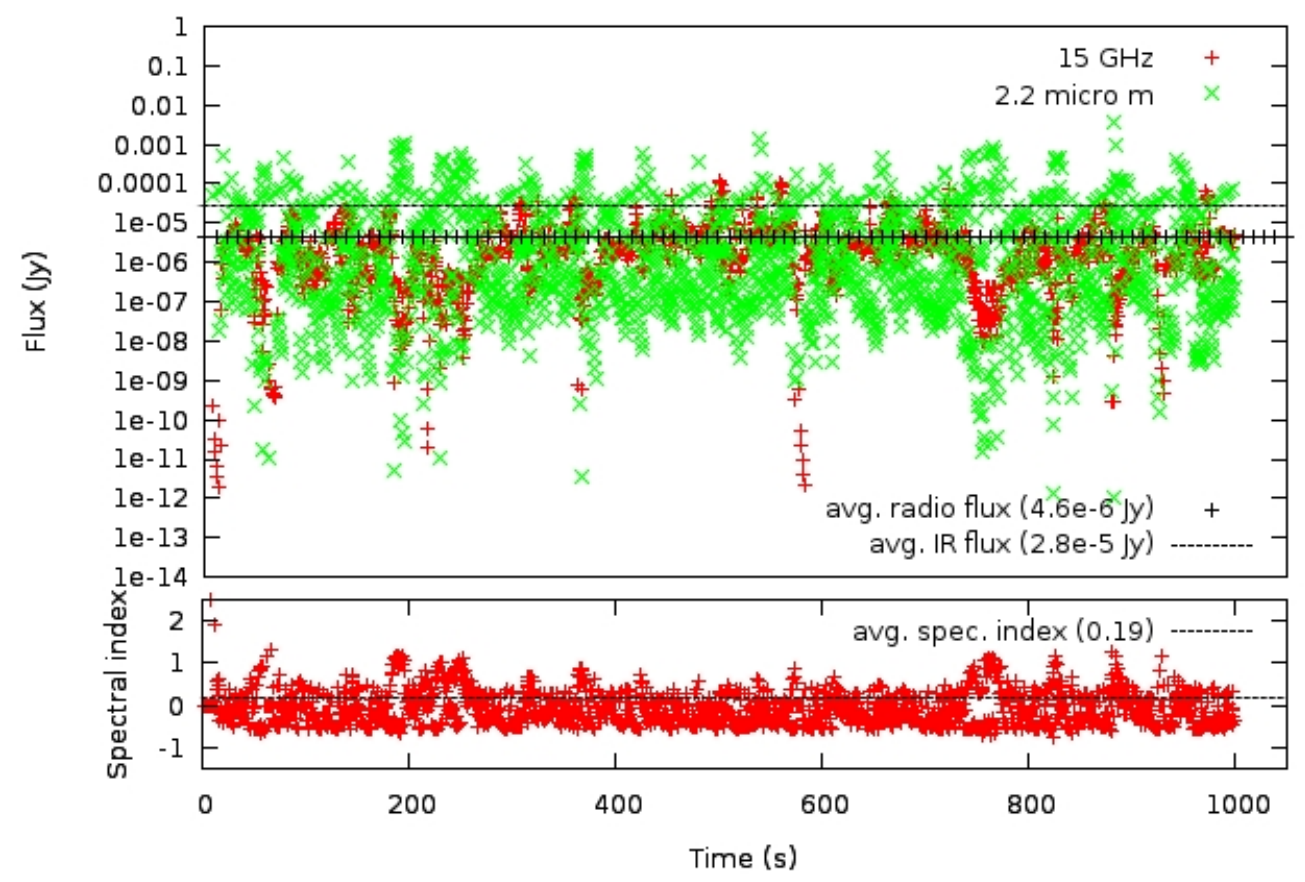

Figure 1: Radio and infra-red lightcurve from the entire jet. The average fluxes and spectral index are shown with straight lines.

Figure 1 shows the radio and infra-red lightcurve from the entire jet. The lightcurves not only shows how the infra-red and radio flux vary with time, but also the spectral index. It can be seen that the spectral index oscillates between $\alpha>0$ (inverted) and $\alpha<0$. The spectral index can also be seen to spend time in the flat spectrum $(\alpha=0)$ regime. Figure 2 shows the spectrum from jet a with almost identical parameters as used for figure 1, higher kinetic luminosity (the parameters are summarized in table 1). It can be seen in figure 2 that IR flux is much higher than radio, giving rise to an overall inverted spectrum. The possible reasons are discussed in the next section. For completeness figure 3 shows a lightcurve from a weak jet. This is primarily to show that with low energy densities, the shell are almost instantly optically thin to radio and hence the radio flux is greater than the IR flux.

The key parameters giving rise to the spectra are summarized in table 1. The injection frequency is the average time gap between shell injections and $\gamma_{\max }$ is the integration upper limit for powerlaw electron distribution (equation 2.11). Similarly $\Gamma_{\max }$ is the upper limit for BLF sampling range. 


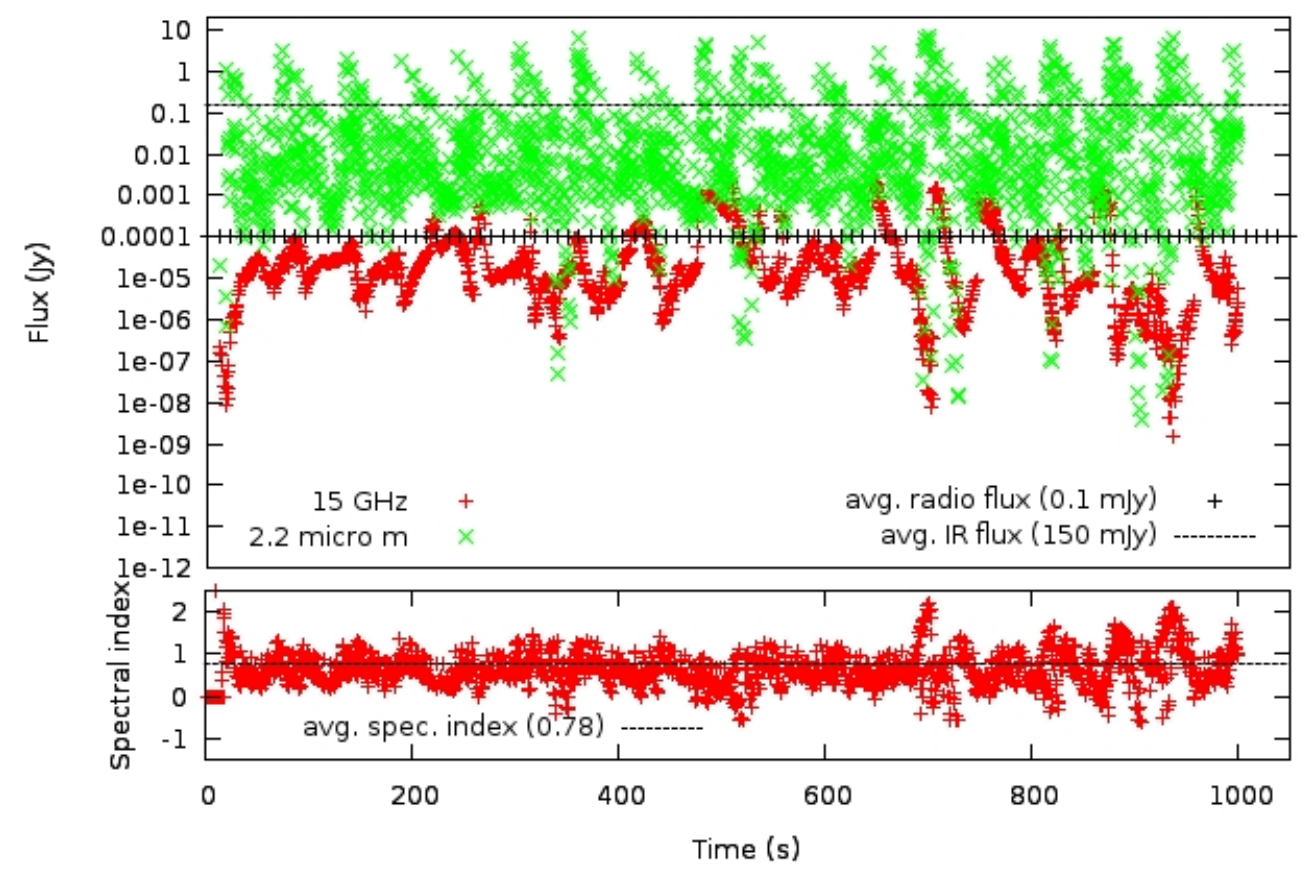

Figure 2: Spectrum showing IR flux much greater than the Radio flux. Average flux and spectral index values are also shown.

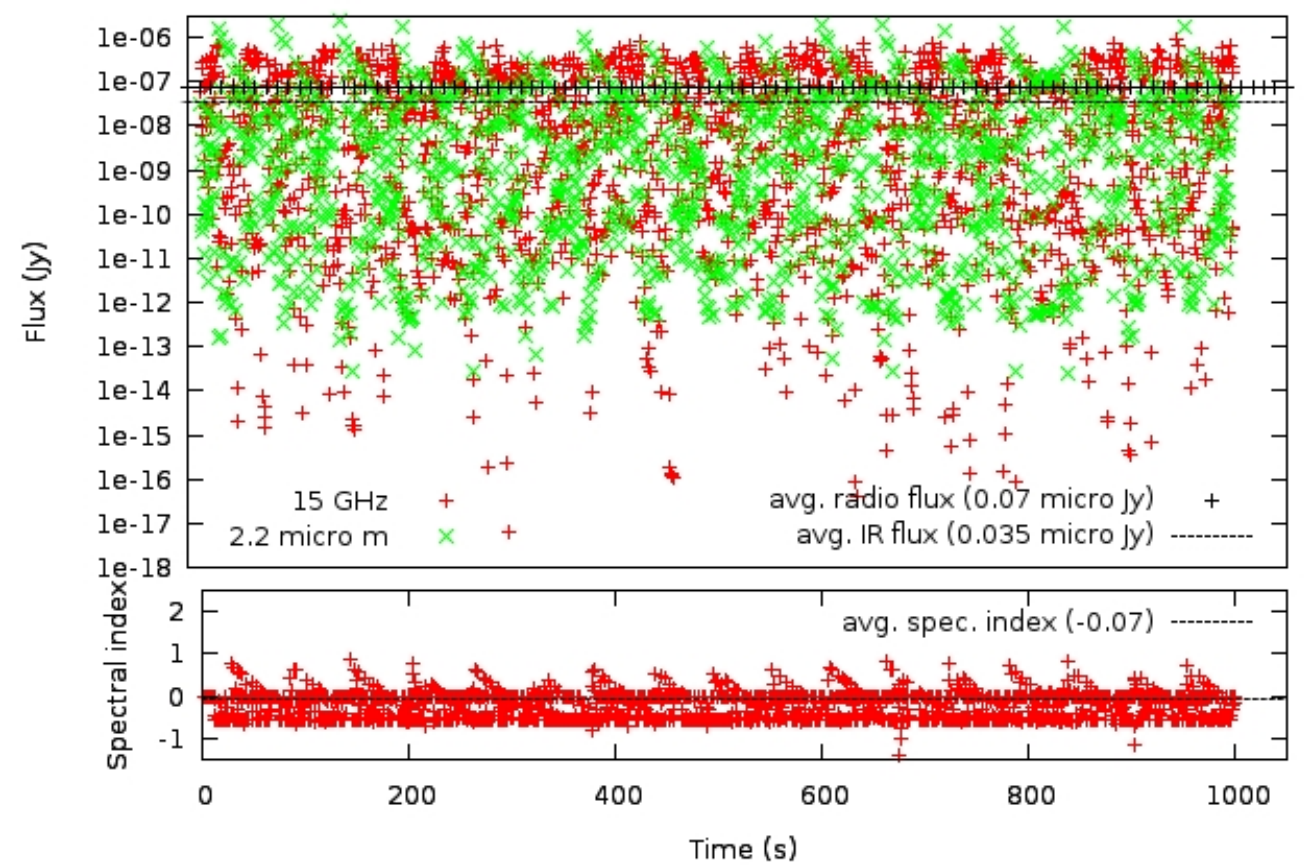

Figure 3: A lightcurve form a weak jet. Average flux and spectral index values are also shown. 
Table 1: Model parameters to achieve the spectra shown (see Results section for details)

\begin{tabular}{llll}
\hline Parameters & Figure 1 & Figure 2 & Figure 3 \\
\hline Jet kinetic luminosity $\left(\mathrm{erg} \mathrm{s}^{-1}\right)$ & $1 \times 10^{35}$ & $1 \times 10^{37}$ & $1 \times 10^{33}$ \\
Distance $(\mathrm{kpc})$ & 2 & 2 & 2 \\
Injection freq. $(\mathrm{Hz})$ & 1 & 1 & 1 \\
$u_{B}$ & 0.1 & 0.1 & 0.1 \\
$u_{e}$ & 0.1 & 0.1 & 0.1 \\
$u_{\text {th }}$ & 0.8 & 0.8 & 0.8 \\
$\gamma_{\max }$ (electrons) & $1 \times 10^{6}$ & $1 \times 10^{6}$ & $1 \times 10^{6}$ \\
$\Gamma_{\max }$ & 2 & 2 & 1.5 \\
Shell width $(\mathrm{m})$ & $1 \times 10^{2}$ & $1 \times 10^{2}$ & $1 \times 10^{2}$ \\
\hline
\end{tabular}

\section{Discussion}

We saw in the results section that with exactly the same parameters except the jet luminosity we get very different lightcurves. This can can be explained by the fact that for the same number of shells, increasing the jet kinetic luminosity increases the mass per shell. This in turn has an influence on the energy generated at each collision i.e. greater mass shell will have a larger energy density. The energy density is the driving force behind the optical depth for a given frequency in the shells and thus giving rise to a different spectrum. In short, more massive shells remain optically thick to radio frequencies for longer and thus completely changing the overall flux from the jet.

If we loook at figure 3, we see that having a weaker jet and thus lower energy density for each shell leads to most shells becoming optically thin to radio very quickly and thus radio flux overall being greater than the IR flux. This can be understood by looking at the position of the self absorbed synchrotron spectrum peak relative to the frequency in question [7].

For the most part $\Gamma_{\max }$ was kept constant, except in the case of spectrum in figure $3 . \Gamma_{\max }$, jet kinetic luminosity and shell width have a similar effect on the overall flux. The influence of these parameters is almost degenerate in the sense that they influence the energy density of the shells. For two colliding shells, greater the difference in their $\Gamma$ s the greater the amount of internal energy generated.

It should be noted that $u_{t h}$ has been set high in the above simulations in order to facilitate the longitudinal shell expansion. This expansion should cause the shells to become optically thin to radio quicker. We see that the fluxes shown in all the spectra are not in very good agreement with the observations for astrophysical systems with similar parameter values. In the case of radio flux especially it is highly non-trivial to obtain a flat spectrum with high fluxes. This indeed an interesting problem and as noted earlier difficult to solve without making certain assumption either about the re-acceleration mechanism [1] or the jet geometry [6]. In our model, with many collisions taking place all along the jet, the shells are continually compressed and powerlaw distributions completely replaced thus becoming optically thick to radio. Shells then have to go through adiabatic expansion in order to become optically thin to radio. However, the adiabatic energy losses mean that the radio flux is significantly lower. We are currently exploring the parameter space that may lead to this 
somewhat fine balance between adiabatic losses and high radio flux for a flat spectrum.

Although it was not shown in these proceedings, the ejection frequency of the shells also plays an important role in the lightcurve and spectra obtained from the jet. The ejection frequency set at $1 \mathrm{~Hz}$ seems to give reasonable results and we not that it is approximately the break frequency in $\mathrm{X}$-ray power spectra of black holes in bright hard state (e.g. Cygnus X-1).

The flaring seen in both radio and IR lightcurves is influenced by various parameters. The flaring behaviour is determined by the balance between how quickly the shells lose their energy via adiabatic losses and the re-energization/compression due to collisions. Rapid adiabatic losses (i.e. larger fraction of energy given to $u_{t h}$ ) causes greater flaring in the lightcurve.

\section{Future directions}

As mentioned in the previous section, our initial aim is to explore the parameter space that gives rise to the Radio-IR flat spectrum. This is an attempt to break the degeneracy present in some of the parameters. Once we have a reasonable picture of what conditions are required to give rise a flat spectrum, we can look at how X-ray variability maybe the driving force behind the shell ejection variability. We also aim to add other physical process to the model such as synchrotron self Compton radiation and radiative losses for the electrons.

\section{References}

[1] R. D. Blandford and A. Konigl, Relativistic jets as compact radio sources, The Astrophysical Journal 232, 34-48 (1979).

[2] R. D. Blandford and C. F. McKee, Fluid dynamics of relativistic blast waves, Physics of Fluids 19 , 1130-1138 (1976).

[3] S. Corbel and R. P. Fender, Near-Infrared Synchrotron Emission from the Compact Jet of GX 339-4, The Astrophysical Journal 573, L35-L39 (2002) [arXiv:astro-ph/0205402].

[4] R. P. Fender, G. G. Pooley, P. Durouchoux, R. P. J. Tilanus, and C. Brocksopp, The very flat radio-millimetre spectrum of Cygnus X-1, Mon. Not. R. Astron. Soc. 312, 853-858 (2000) [arXiv:astro-ph/9910184].

[5] R. M. Hjellming and K. J. Johnston, Radio emission from conical jets associated with X-ray binaries, The Astrophysical Journal 328, 600-609 (1988).

[6] C. R. Kaiser, The flat synchrotron spectra of partially self-absorbed jets revisited, Mon. Not. R. Astron. Soc. 367, 1083-1094 (2006) [arXiv: astro-ph/ 0601103 ].

[7] M. S. Longair, High energy astrophysics. Vol.2: Stars, the galaxy and the interstellar medium, Cambridge: Cambridge University Press, lc1994, 2nd ed., 1994.

[8] M. Spada, G. Ghisellini, D. Lazzati, and A. Celotti, Internal shocks in the jets of radio-loud quasars, Mon. Not. R. Astron. Soc. 325, 1559-1570 (2001) [arXiv: astro-ph/ 0103424 ]. 